\title{
Metal abundances and kinematics of quasar absorbers
}

\section{Absorption systems toward J2233-606^}

\author{
S. A. Levshakov ${ }^{1, \star \star}$, I. I. Agafonova ${ }^{2}$, M. Centurión ${ }^{3}$, and I. E. Mazets ${ }^{2}$ \\ 1 Division of Theoretical Astrophysics, National Astronomical Observatory, Mitaka, Tokyo 181-8588, Japan \\ 2 Department of Theoretical Astrophysics, Ioffe Physico-Technical Institute, 194021 St. Petersburg, Russia \\ 3 Osservatorio Astronomico di Trieste, Via G. B. Tiepolo 11, 34131 Trieste, Italy
}

Received 4 October 2001 / Accepted 18 December 2001

\begin{abstract}
The metal line profiles of different ions observed in high $\mathrm{H}$ i column density systems $\left[N(\mathrm{H} \mathrm{I})>10^{16} \mathrm{~cm}^{-2}\right]$ in quasar spectra can be used to constrain the ionization structure and kinematic characteristics of the absorbers. For these purposes, a modified Monte Carlo Inversion (MCI) procedure was applied to the study of three absorption systems in the spectrum of the HDF-South quasar J2233-606 obtained with the UVES spectrograph at the VLT/Kueyen telescope. The MCI does not confirm variations of metal abundances within separate systems which were discussed in the literature. Instead, we found that an assumption of a homogeneous metal content and a unique photoionizing background is sufficient to describe the observed complex metal profiles. It was also found that the linear size $L$ and the line-of-sight velocity dispersion $\sigma_{\mathrm{v}}$ measured within the absorbers obey a scaling relation, namely, $\sigma_{\mathrm{v}}$ increases with increasing $L$, and that metal abundance is inversely proportional to the linear size of the system: the highest metallicity was measured in the system with the smallest $L$.
\end{abstract}

Key words. cosmology: observations - line: formation - line: profiles - galaxies: abundances - quasars: absorption lines - quasars: individual: J2233-606

\section{Introduction}

Absorption systems in quasar spectra provide unique information on the intervening intergalactic matter (IGM) up to redshift $z \simeq 6$, back to the time when the Universe was less than $7 \%$ of its present age. High resolution spectroscopic observations available nowadays at large telescopes open new opportunities to investigate the physical nature of quasar absorbers. Reliable data on the chemical composition of the IGM and on the physical characteristics (like velocity and density distributions, volumetric gas density, kinetic temperature, ionization structure etc.) of the absorbers is an important clue to our understanding of galaxy formation, chemical evolution of the IGM and the origin of the large-scale structure.

Send offprint requests to: S. A. Levshakov,

e-mail: lev@yso.mtk.nao.ac.jp

* Based on public data released from UVES Commissioning at the VLT Kueyen telescope (ESO, Paranal, Chile), the New Technology Telescope (ESO, La Silla, Chile) and the NASA/ESA Hubble Space Telescope obtained at the Space Telescope Science Institute, which is operated by the Association of Universities of Research in Astronomy, Inc. under NASA contract NAS 5-26555.

** On leave from the Ioffe Physico-Technical Institute, Russian Academy of Sciences, St. Petersburg.
In recent investigations much attention find the metal systems which are the absorbers exhibiting as a rule numerous lines of low (like HI, CII, Si II, Mg II, Al II) and high (like C III, N III, Si III, C IV, Si IV, N v) ionized species.

Presence of metals provides a unique opportunity to study the physical conditions of matter at early epochs. Unfortunately, the computational methods usually applied to high resolution spectra lie quite often behind the quality of observational data and fail to extract from them all encoded information. The common processing method consists of the deconvolution of complex absorption profiles into an arbitrary number of separate components (assuming a constant gas density within each of them) which are then fitted to Voigt profiles. However, in many cases this procedure may not correspond to real physical conditions: observed complexity and non-similarity of the profile shapes of different ions indicate that these systems are in general absorbers with highly fluctuating density and velocity fields tightly correlated with each other. Too high or too low gas temperatures, extremely varying metallicities between subcomponents, exotic UV background spectra and other physically badly founded outcomings may be artifacts arising from the inconsistency of the applied methods (see examples in Levshakov et al. 1999; Levshakov et al. 2000b, hereafter Paper I). 
In Paper I we developed a new method for the QSO spectra inversion, - the Monte Carlo Inversion (MCI), - assuming that the absorbing region is a cloud with uniform metallicity but with fluctuating density and velocity fields inside it. This computational procedure which is based on stochastic optimization allows us to recover both the underlying hydrodynamical fields and the physical parameters of the gas. First application of the MCI to the analysis of the $z_{\text {abs }}=3.514$ system toward Q08279+5255 (Levshakov et al. 2000a) has shown that the proposed method is very promising especially in the inversion of complex absorption spectra with many metal lines.

In this paper we start a new comprehensive survey of the metal systems for which high resolution and high signal-to-noise spectra are available. We present here the results for three absorption systems $\left(z_{\mathrm{abs}}=1.87,1.92\right.$ and 1.94) from the spectrum of the quasar J2233-606 which have been already studied by Prochaska \& Burles (1999), and D'Odorico \& Petitjean (2001, hereafter DP) using the common Voigt fitting method. We re-calculate these systems using the MCI in order to compare the applicability of both approaches and to show up their restrictions.

The structure of the paper is as follows. In Sect. 2 the data sets used in the MCI analysis are described. Section 3 contains the details of the applied computational procedure. The results obtained for each of the mentioned above systems are presented in Sect. 4. Conclusions are reported in Sect. 5. In Appendix the general equation of the entropy production rate is given which is used to calculate the density and velocity configurations along the line of sight exhibiting minimum dissipation.

\section{Data}

High-quality data of the Hubble Deep Field South (HDFS) quasar J2233-606 ( $\left.z_{\mathrm{em}}=2.238, B \simeq 17.5\right)$ were obtained during the commissioning of the UVES on the VLT $8.2 \mathrm{~m}$ Kueyen telescope at Paranal (Chile) in October 1999. The resolving power at which the spectra were recorded in the spectral range $\lambda \lambda=3050-10000 \AA$ was $R \simeq 45000$, corresponding to velocity resolution of $F W H M \simeq 6.7 \mathrm{~km} \mathrm{~s}^{-1}$.

The data reduction and the identification of metal absorption-line systems in the J2233-606 spectrum are reported in Cristiani \& D'Odorico (2000). In our study we also used the J2233-606 echelle spectrum $(R \simeq 30000$, $\lambda \lambda 2275-3118 \AA$ ) obtained with the HST/STIS (Savaglio 1998).

\section{Computational procedure}

The complete description of our computational procedure is given in Paper I. Here we summarize briefly basic model assumptions and emphasize new details recently included in the MCI.

We assume that all lines observed in a metal system arise in a continuous absorbing gas slab of a thickness
$L$ (presumably the outer region of a foreground distant galaxy). The absorber exhibits a fluctuating gas density and a mixture of bulk motions such as infall and outflows, tidal flows etc., resulting in a stochastic velocity field. Metal abundances are assumed to be constant within the absorber and gas is supposed to be optically thin for the ionizing UV radiation.

Within the absorbing region the radial velocity $v(s)$ and total hydrogen density $n_{\mathrm{H}}(s)$ distributions along the line of sight are the same for all ions. In the computational procedure these two random fields are represented by their sampled values at equally spaced intervals $\Delta x(x$ is dimensionless radial coordinate $s / L)$, i.e. by the vectors $\left\{v_{1}, \ldots, v_{k}\right\}$ and $\left\{n_{1}, \ldots, n_{k}\right\}$ with $k$ large enough to describe the narrowest components of complex spectral lines.

Further we suppose the thermodynamic and ionization equilibrium at each computational point along the sightline which means that fractional ionizations of different ions are determined exclusively by the gas density and vary from point to point. These fractional ionization variations are just the cause of the observed diversity of profile shapes. To calculate the kinetic temperature and fractional ionization of ions the photoionization code CLOUDY (Ferland 1997) was used.

The inputs to CLOUDY are the dimensionless ionization parameter $U=n_{\mathrm{ph}} / n_{\mathrm{H}}\left(n_{\mathrm{ph}}-\right.$ the number density of photons with energies above $1 \mathrm{Ry})$, metallicity and the background ionizing spectrum for which the HaardtMadau spectrum (HM) was adopted (Haardt \& Madau 1996). The number density of the ionizing photons for this spectrum

$n_{\mathrm{ph}}=\frac{4 \pi}{c h} J_{912} \int_{\nu_{\mathrm{c}}}^{\infty}\left(\frac{J_{\nu}}{J_{912}}\right) \frac{\mathrm{d} \nu}{\nu}$,

equals at $z=2$ to $2.26 \times 10^{-5} \mathrm{~cm}^{-3}$ [here $c, h, \nu_{\mathrm{c}}$ and $J_{\nu}$ are the speed of light, the Planck constant, the frequency of the hydrogen Lyman edge, and the specific intensity (in $\operatorname{ergs} \mathrm{cm}^{-2} \mathrm{~s}^{-1} \mathrm{sr}^{-1} \mathrm{~Hz}^{-1}$ ) at frequency $\nu$, respectively].

Fractional ionization curves $\Upsilon(U)$ were computed with CLOUDY for solar abundance pattern and different metallicities and then included in the MCI code to calculate the optical depths for ions involved in the fitting. If the obtained solution revealed the abundance pattern different from the solar one, $\Upsilon(U)$ were recalculated for this new pattern and all computations repeated. It should be noted, however, that differences of $0.2 \div 0.3$ dex from solar values influence the fractional ionizations only weakly.

The values of velocity and density at subsequent computational points are considered to be correlated and are described by means of Markovian processes. In particular, the velocity is computed as follows:

$v(x+\Delta x)=f_{\mathrm{v}} v(x)+\epsilon(x+\Delta x)$,

where $f_{\mathrm{v}}=R_{\mathrm{v}}(\Delta x) / \sigma_{\mathrm{v}}^{2}, R_{\mathrm{v}}$ being the correlation between the velocity values at points separated by a distance $\Delta x$, i.e. $R_{\mathrm{v}}=\langle v(x+\Delta x) v(x)\rangle, \sigma_{\mathrm{v}}$ the velocity dispersion of 

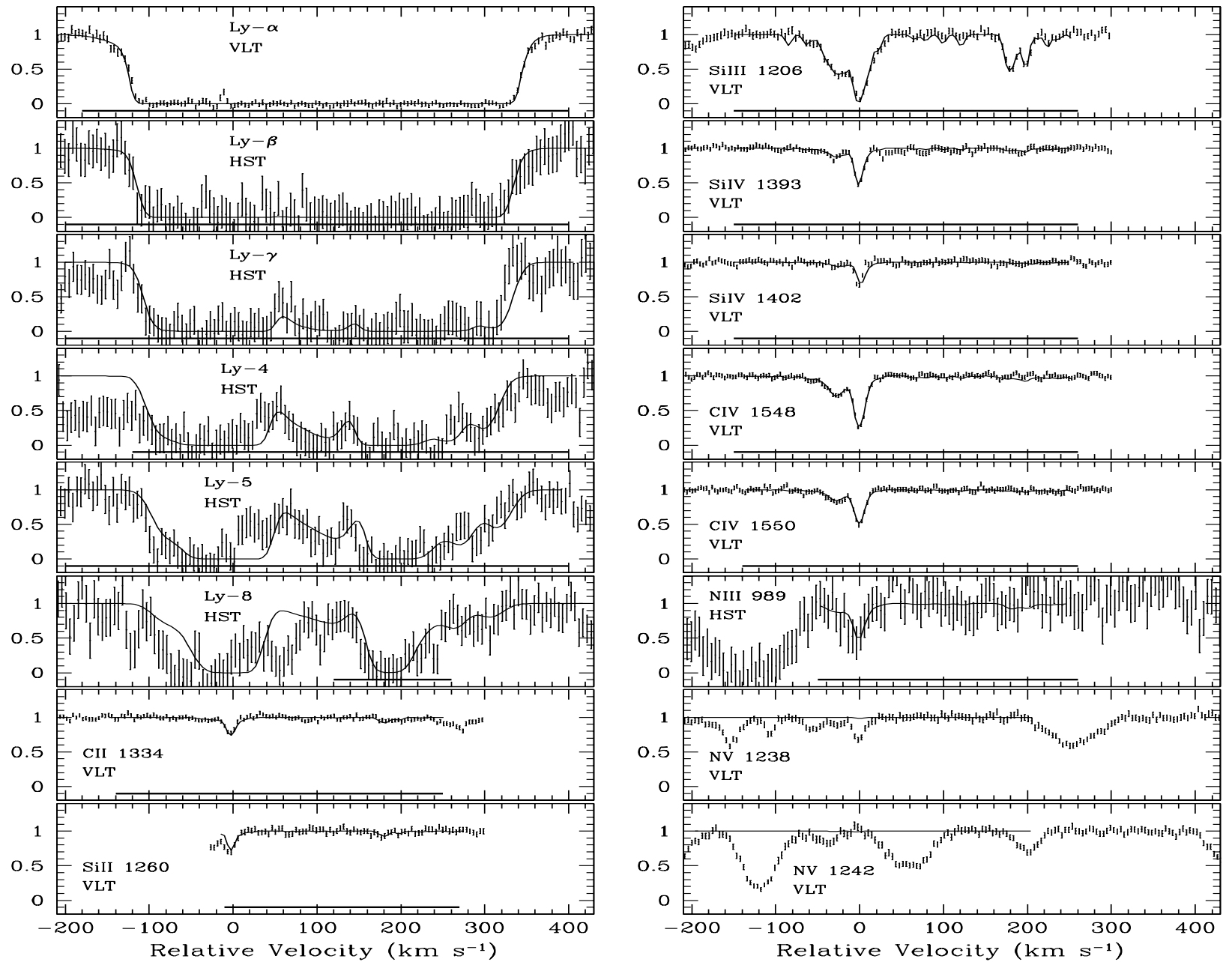

Fig. 1. Hydrogen and metal absorption lines associated with the $z_{\text {abs }} \simeq 1.92$ system toward J2233-606 (normalized intensities are shown by dots with $1 \sigma$ error bars). The zero radial velocity is fixed at $z=1.92595$. Smooth lines are the synthetic spectra convolved with the corresponding point-spread functions $\left(F W H M_{\mathrm{VLT}}=6.7 \mathrm{~km} \mathrm{~s}^{-1}, F W H M_{\mathrm{HST}}=10.0 \mathrm{~km} \mathrm{~s}{ }^{-1}\right)$ and computed with the physical parameters from Table 1. Bold horizontal lines mark pixels included in the optimization procedure. The normalized $\chi_{\min }^{2}=1.10$ (the number of degrees of freedom $\nu=1450$ ).

bulk motions, and $\epsilon$ a random normal variable with zero mean and dispersion

$\sigma_{\epsilon, \mathrm{v}}=\sigma_{\mathrm{v}} \sqrt{1-f_{\mathrm{v}}^{2}}$

Density is supposed to have a log-normal distribution (characterized by the mean $n_{0}$ and the second central moment $\sigma_{\mathrm{y}}$ of dimensionless variable $\left.y=n_{\mathrm{H}} / n_{0}\right)$ and is described with help of an auxiliary Markovian process $\nu(x)$ :

$\nu(x+\Delta x)=f_{\nu} \nu(x)+\epsilon(x+\Delta x)$,

where $f_{\nu}=R_{\nu}(\Delta x) / \sigma_{\nu}^{2}, R_{\nu}$ being the correlation between the values of $\nu$ at points separated by a distance $\Delta x, \sigma_{\nu}$ the logarithmic density dispersion, $\sigma_{\nu}=\sqrt{\ln \left(1+\sigma_{\mathrm{y}}^{2}\right)}$ and $\epsilon$ a random normal variable with zero mean and dispersion $\sigma_{\epsilon, \nu}=\sigma_{\nu} \sqrt{1-f_{\nu}^{2}}$.

Having defined $\nu(x)$, the total hydrogen density can be obtained as

$n_{\mathrm{H}}(x)=n_{0} \mathrm{e}^{\nu(x)-\frac{1}{2} \sigma_{\nu}^{2}}$.
The optical depth $\tau_{\mathrm{a}, i}(\lambda)$ at wavelength $\lambda$ for element 'a' in $i$ th ionizing stage is calculated from the equation:

$$
\begin{aligned}
& \tau_{\mathrm{a}, i}(\lambda)=k_{0} Z_{\mathrm{a}} N_{0} \mathrm{e}^{-\frac{1}{2} \sigma_{\nu}^{2}} \\
& \quad \times \int_{0}^{1} \mathrm{e}^{\nu(x)} \Upsilon_{\mathrm{a}, i}[U(x)] \Phi_{\lambda}\left[\Delta \lambda_{\mathrm{D}}(x), v(x)\right] \mathrm{d} x,
\end{aligned}
$$

where $k_{0}$ is a constant, $N_{0}=n_{0} L$ - the expectation value of the total hydrogen column density, $Z_{\mathrm{a}}=n_{\mathrm{a}} / n_{\mathrm{H}}$ - the relative abundance of element "a", $\Upsilon_{\mathrm{a}, i}[U(x)]$ - the fractional ionization for stage $i, \Phi_{\lambda}$ - the profile function, $\Delta \lambda_{\mathrm{D}}$ - the Doppler width of the line.

Before being compared with the observed spectrum, the synthetic intensities $\exp [-\tau(\lambda)]$ are convolved with the spectrograph point-spread function.

Thus the proposed model is fully defined by specifying the following values: the velocity vector $\left\{v_{i}\right\}$, the total hydrogen density vector $\left\{n_{i}\right\}$, the total hydrogen column density $N_{0}$, the mean ionization parameter $U_{0}$, the radial velocity dispersion $\sigma_{\mathrm{v}}$, the density second central moment $\sigma_{\mathrm{y}}$, the element abundances $Z_{\mathrm{a}}$ and the correlation 


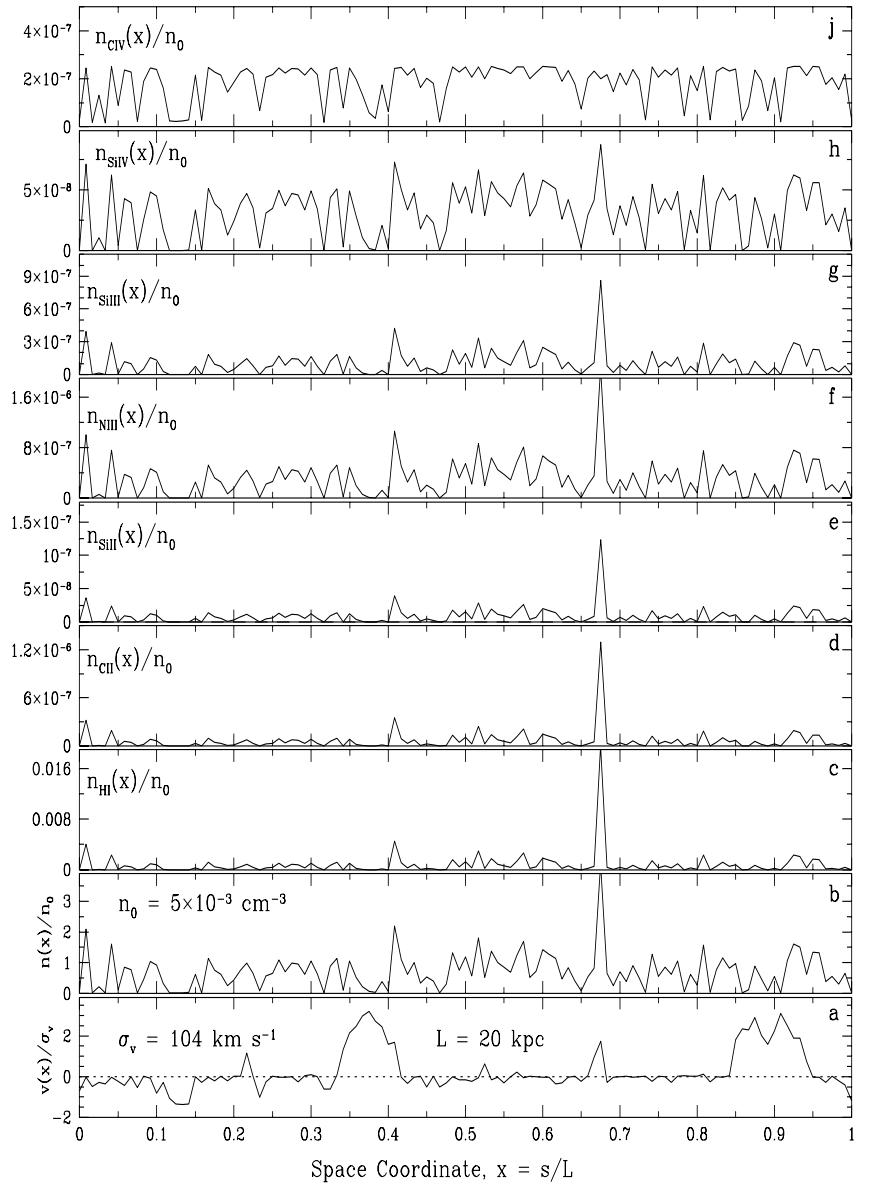

Fig. 2. Computed velocity (panel a) and density of gas (panel $\mathbf{b}$ ) and ions (panels $\mathbf{c}-\mathbf{j}$ ) distributions along the line of sight for the system at $z=1.92595$ toward J2233-606. Shown are patterns rearranged according to the principle of minimum entropy production rate (see text).

coefficients $f_{\mathrm{v}}$ and $f_{\nu}$. The common least-squares minimization (LSM) of the objective function is used to estimate the model parameters ${ }^{4}$.

The computational procedure itself consists of two steps: firstly a point in the parameter space $\left(N_{0}, U_{0}, \sigma_{\mathrm{v}}, \sigma_{\mathrm{y}}, Z_{\mathrm{a}}\right)$ is chosen and then an optimal configuration of $\left\{v_{i}\right\}$ and $\left\{n_{i}\right\}$ for this parameter set is searched for. Correlation coefficients are considered as external parameters and remain fixed during the calculations.

The optimization of $\left\{v_{i}\right\}$ and $\left\{n_{i}\right\}$ is the most timeconsuming part of the procedure and needs an effective algorithm to achieve a quick and stable convergence of the computations. In the MCI we use the simulated annealing with Tsallis acceptance rule (Xiang et al. 1997) and an adaptive annealing temperature choice. Namely, the

\footnotetext{
${ }^{4}$ The LSM is computationally identical to the standard $\chi^{2}$ minimization, but it can be used to evaluate the uncertainties of the fitting parameters even in cases of correlated measurements. One must realize that the rebinning used in the data reduction procedure introduces a correlation between data point values (Levshakov et al. 2002).
}

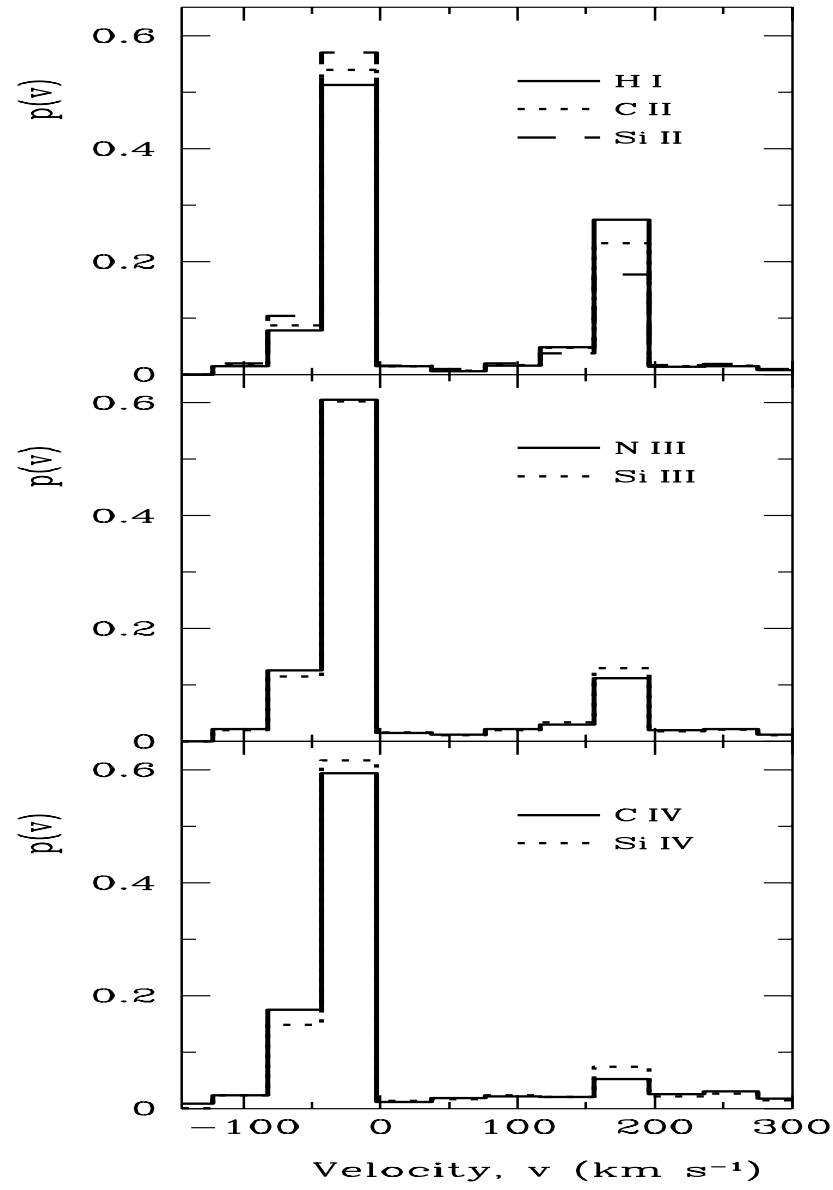

Fig. 3. Density-weighted velocity distribution functions, $p(v)$, for Hi, CiI, Si II, N III, Si III, C IV, and Si IV as restored by the MCI procedure in the $z=1.92595$ system toward J2233-606.

annealing temperature $T_{\mathrm{a}}$ at iteration $k+1$ is decreased according to following equation:

$T_{\mathrm{a}}^{k+1}=\frac{T_{\mathrm{a}}^{k}}{1+\beta \frac{\Delta E_{k}}{E_{k}} r}$,

where $\Delta E_{k} / E_{k}$ is the relative energy ( $\equiv$ the sum of squares of the deviations, $\mathcal{S}$ ) variation on step $k, r$ is the acceptance rate, i.e. the ratio of the accepted trials to the total trial number, and $\beta$ is a constant of order 1.

The calculation of the uncertainty ranges for the fitting parameters is in our method not so straightforward as a simple inversion of the Hesse matrix since the velocity and density distributions represent additional degrees of freedom and widen, in general, the confidence intervals. However, these $\left\{v_{i}\right\}$ and $\left\{n_{i}\right\}$ distributions themselves are nuisance parameters and should be "integrated out" when one computes the errors for the other parameters. To estimate the confidence levels, the following procedure can be applied: the values of the physical parameters in the vicinity of the global minimum of the objective function are chosen at random and then the optimal density and velocity distributions are computed. Assuming that the probability of each parameter set can be linked to the derived $\mathcal{S}$ value $[$ e.g. as $\exp (-\mathcal{S})]$ we can estimate from the 

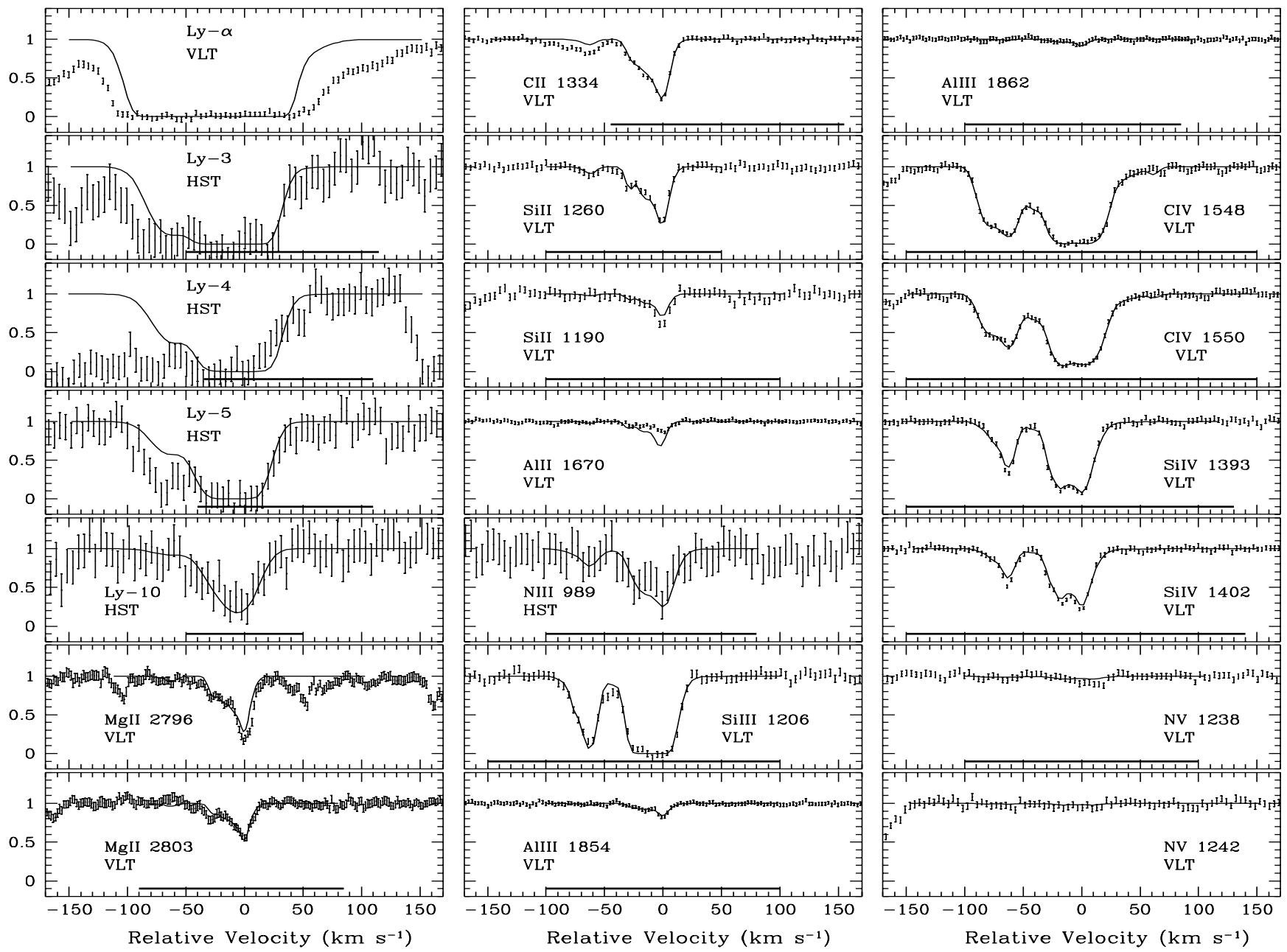

Fig. 4. Same as Fig. 1 but for the $z_{\text {abs }} \simeq 1.94$ system toward J2233-606. The zero radial velocity is fixed at $z=1.942616$. The corresponding physical parameters are listed in Table 1 . The normalized $\chi_{\min }^{2}=1.08$ (the number of degrees of freedom $\nu=888)$.

obtained sample the joint probability density function for parameters and hence calculate all necessary statistical moments.

It should be noted, however, that the reliable estimation of this multidimensional function requires a very large sample which is quite time consuming. In our case the exact estimation of confidence levels is not very crucial taking into account the intrinsic uncertainties in atomic data (e.g. Savin 2000) or unknown shape and intensity of the local background ionizing radiation. Because of this we restricted our samples to a few dozens of points and estimated the accuracy of the fitting parameters only approximately.

The recovered density and velocity patterns are not unique - many configurations are possible with comparable probability. But all these configurations have the same density-weighted velocity distributions which actually determine the observed line shapes (see Paper I). As already mentioned above, we represent these random fields by their values sampled at equally spaced intervals $\Delta x$. In order to compare the calculated patterns we rearrange these values in such a way that the final configuration exhibits a lowest rate of entropy production: according to the Prigogine theorem (Prigogine 1967), this configuration has the minimal dissipation and, hence, is more stable and more probable. All necessary equations to calculate the entropy production are presented in Appendix. We stress, however, that configurations produced on the base of such rearrangement should in no case be considered as something final - they represent only the (most) probable case of the density and velocity distributions along the line of sight and are used here exclusively for illustrations.

\section{Results on individual systems}

\subsection{J2233-606, $z_{a b s} \simeq 1.92$}

The system at $z_{\text {abs }} \simeq 1.92$ toward the quasar J2233-606 has saturated hydrogen lines (from Ly- $\alpha$ up to Ly-8) and metal lines of C II, Si II, Si III, C IV, and Si IV. The results obtained with the MCI are presented in Table 1 and illustrated in Figs. 1 and 2. Parts of profiles included in the $\chi^{2}$ minimization are marked by horizontal lines at the panel bottoms in Fig. 1. Profiles of the doublet N v $\lambda 1238 \AA$ and 


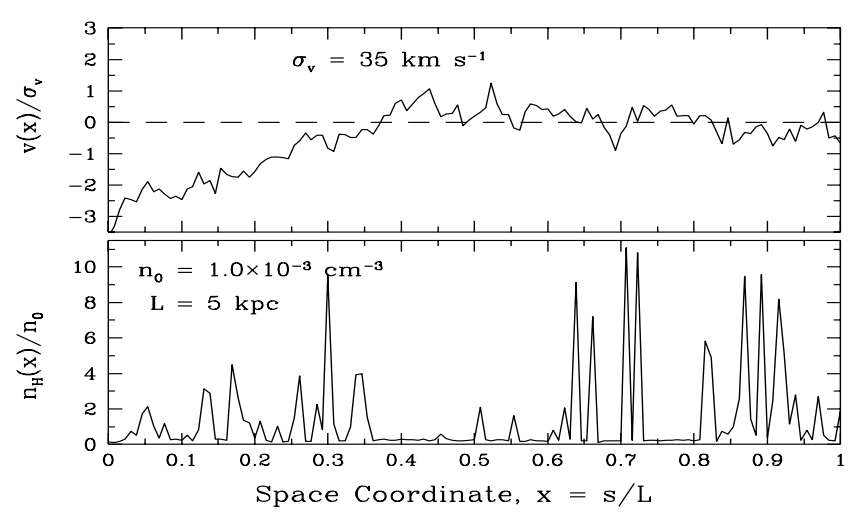

Fig. 5. Computed velocity (upper panel) and gas density (lower panel) distributions along the line of sight for the system at $z=1.942616$ toward J2233-606. Shown are patterns rearranged according to the principle of minimum entropy production rate (see text).

N v $\lambda 1242 \AA$ were calculated later using the obtained velocity and density distribution and the metallicity derived from the fitting of N III $\lambda 989 \AA$. It is seen from Fig. 1 that most spectral features can be well represented assuming uniform metallicities and a common HM UV background. Figure 2 demonstrates the distribution of the radial velocity and gas density (panels a and b) along the line of sight (rearranged in accord with the principle of minimal entropy production rate). The density distributions for the ions involved in the optimization are shown in panels $\mathrm{c}-\mathrm{j}$, whereas the density-weighted velocity distributions which determine the shapes of the spectral lines are presented in Fig. 3. This figure shows that the density-weighted velocity distributions for low ions CII and Si II are similar, but differ from those for high ions C IV and Si IV. These distributions easily explain why the lines of C II and Si II look very much alike and why their centers are displaced by $\Delta v \simeq 4 \mathrm{~km} \mathrm{~s}^{-1}$ (DP) with respect to C IV and Si IV.

The study of this system by DP, who used the standard Voigt profile fitting, produced comparable column densities (albeit 20-50\% smaller). However, the metallicities obtained by DP for two main clouds (at $v=0 \mathrm{~km} \mathrm{~s}^{-1}$ and $v=-43 \mathrm{~km} \mathrm{~s}^{-1}$ ) differ nearly by two orders of magnitude: $[\mathrm{X} / \mathrm{H}]=-0.9$ and -2.7 , respectively (in our case $[\mathrm{X} / \mathrm{H}] \simeq-2.2$ for the whole system). As shown in Paper $\mathrm{I}$, the Voigt fitting may in general yield correct column densities when applied to unsaturated lines, but the mean $U$ and, hence, the ionization corrections may not be unambiguous. Therefore, the conclusion made by DP that the $z_{\text {abs }} \simeq 1.92$ system contains "a region of intense starformation activity" may not be well justified since this result is model dependent.

The values of the average gas density $n_{0}$ and kinetic temperature $T_{\text {kin }}$, and the cloud thickness $L$ estimated in our model (see Table 1) are typical for the Ly- $\alpha$ systems discussed in the literature (e.g., Giallongo \& Petitjean 1994; Viegas et al. 1999; Prochaska \& Burles 1999; Chen et al. 1998; Chen et al. 2001). Low metallicity for the whole system $([\mathrm{X} / \mathrm{H}]<-2.0)$ and its dimension of $20 \mathrm{kpc}$ imply that this system can originate in a galactic halo or in a large scale structure object.

\section{2. $J 2233-606, z_{a b s} \simeq 1.94$}

This system exhibits a plenty of metal lines in different ionization stages. The metal profiles are not very complex and extend over the velocity range from $-100 \mathrm{~km} \mathrm{~s}^{-1}$ to $100 \mathrm{~km} \mathrm{~s}^{-1}$. Results obtained with the MCI are presented in Table 1 and shown in Figs. 4 and 5. As in the previous system, most absorption features can be well described with uniform metallicities and a common HM spectrum. The Ly- $\alpha$ profile is contaminated by the forest absorption in the blue and red wings and therefore the Ly- $\alpha$ absorption feature was not involved in the analysis. The profiles of MgII $\lambda 2796 \AA$ and Al II $\lambda 1670 \AA$ were computed later using the derived velocity and density distributions. $\mathrm{Mg}$ II $\lambda 2796 \AA$ is contaminated by a telluric line and this explains the difference between the computed and observed profiles. The synthetic and observed profiles of Al II $\lambda 1670 \AA$ show much more pronounced discrepancy. Fractional ionisation curves for $\mathrm{Al}$ II and Al III were computed with CLOUDY. These curves allowed us to fit the $\mathrm{Al}$ III doublet quite well with the $\mathrm{Al}$ abundance similar to that obtained for the other metals. However, when the Al II profile was included in the fitting, the Al metallicity differed by order of magnitude from the other metals. Besides it was impossible to fit adequately the Al III doublet. Similar behaviour of Al was reported also by DP who noted that "the recombination coefficients used to compute the aluminium ionisation equilibrium [in CLOUDY] are probably questionable".

Column densities derived by DP coincide well (within $15 \%$ ) with that obtained in our procedure except for the saturated Si III $\lambda 1206 \AA$ line for which the Voigt fitting gave nearly 2 times lower value. The abundances estimated in DP scatter again from component to component, but nevertheless they conclude that "the gas in this system is likely of quite high metallicity (larger than 0.1 solar)".

Similar to the Voigt fitting, the MCI also delivered for this system high metal abundancies: one third solar for carbon and silicon and nearly two times lower for nitrogen, magnesium and aluminium. Taking into account this result and a compact dimension $(\simeq 5 \mathrm{kpc}$, see Table 1$)$ of the absorbing region we come to the same conclusion as Prochaska \& Burles (1999) did: the system at $z=1.94$ can hardly be a large scale structure object (like a filament or a wall) and should be related to a galactic system (may be a region of intense star formation).

\subsection{J2233-606, $z_{a b s} \simeq 1.87$}

This is the most interesting system from the family of the absorbers at $z=1.9$ toward J2233-606. The metal line profiles show a rather complex structure extending over the velocity range of about $700 \mathrm{~km} \mathrm{~s}^{-1}$. Some of these 

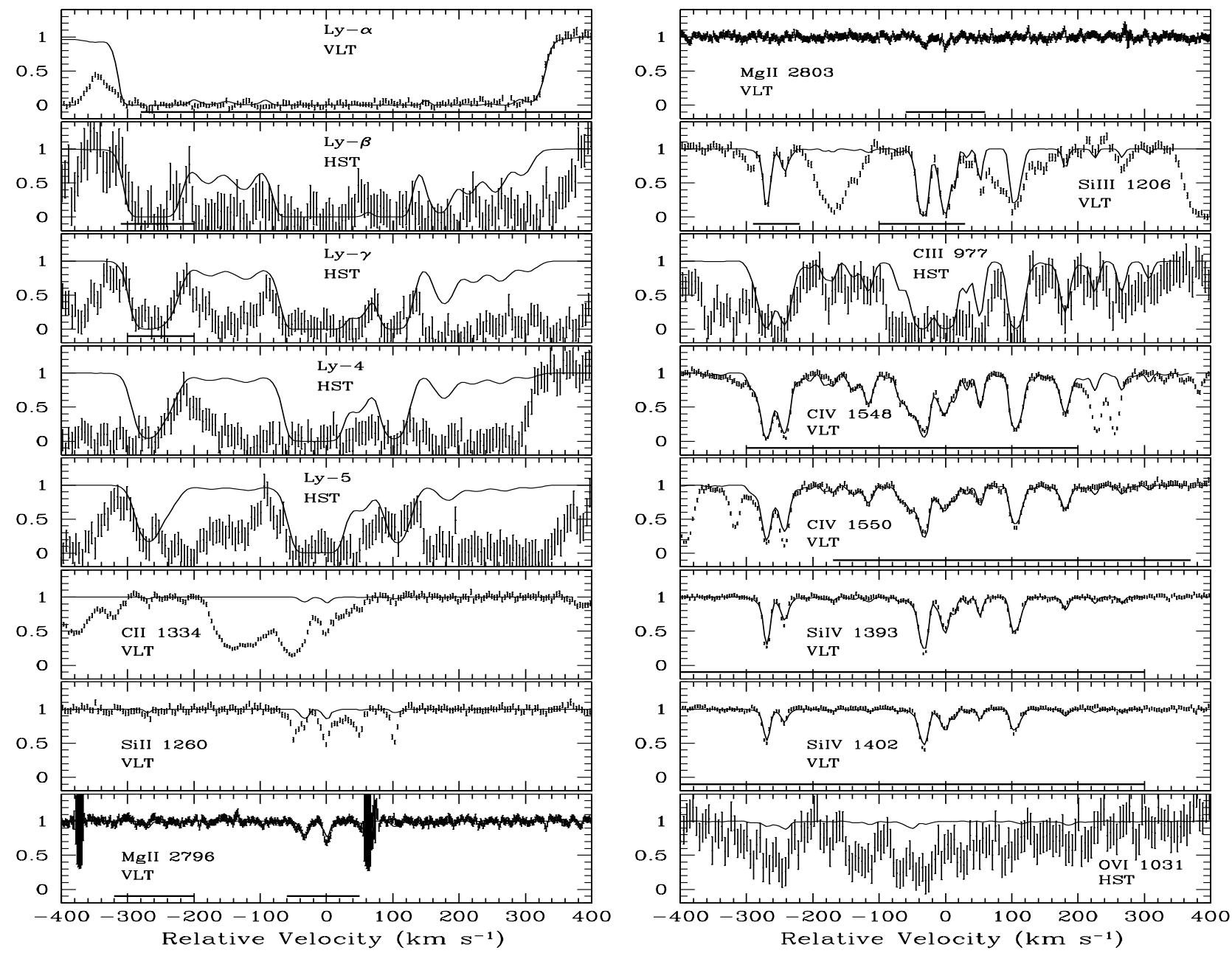

Fig. 6. Same as Fig. 1 but for the $z_{\text {abs }} \simeq 1.87$ system toward J2233-606. The zero radial velocity is fixed at $z=1.87008$. The corresponding physical parameters are listed in Table 1 . The normalized $\chi_{\min }^{2}=1.60$ (the number of degrees of freedom $\nu=1158)$.

profiles are severely blended that hampers the unique Voigt profile deconvolution (e.g. DP assumed 17 components to describe metal profiles).

The MCI code turned out to be much more robust and was able to recover the self-consistent line profiles even under such unfavourable conditions. The physical parameters which the MCI delivered for the $z=1.87$ system together with the underlying velocity and density distributions are presented in Table 1 and in Figs. 6 and 7. It is seen from Fig. 6 that like in the previous two systems all lines are well described with a single parameter set, uniform metallicities and a common HM UV background. The blue wing of the Ly- $\alpha$ line is contaminated by the forest absorption as is clearly seen from the Ly- $\beta$ and Ly$\gamma$ profiles. The synthetic profile of the Ovi $\lambda 1031 \AA$ line was calculated later using the derived best fitting parameters and the oxygen abundance $[\mathrm{O} / \mathrm{H}]=-1.0$ (which is about 3 times over the other element abundances from this system). Even with the increased abundance the synthetic profile of $\mathrm{O}$ VI is still much weaker than the observed intensities. This discrepancy rules out the ionization of
O VI by the adopted background radiation. Taking into account that all other elements have been well described with a given HM spectrum and that the collisional ionization of oxygen can hardly be effective at low densities $\left(n_{\mathrm{H}} \sim 10^{-5}\right)$ and temperatures of $\sim 25000 \mathrm{~K}$, this result seems to favor the interpretation that the $\mathrm{O}$ VI ion and the other ions do not arise in the same gas (Kirkman \& Tytler 1999; Reimers et al. 2001).

According to our results, the absorber at $z=1.87$ could be a large size cloud with very high velocity dispersion. Its estimated linear size of $80 \mathrm{kpc}$ is consistent with dimensions of extended gaseous envelopes observed around galaxies at $z<1$. In these envelopes, Mg II absorption is the dominant observational signature at the distancies up to a few tens of kiloparsecs (Bergeron \& Boissé 1991), whereas highly ionizied species like C IV are observed at distances of at least $100 \mathrm{kpc}$ from galactic centers (Chen et al. 2001). Since the extended structure of the same order of magnitude is observed at $z=1.87$, we may conclude that this system arises in the external halo at large galactocentric distances. 


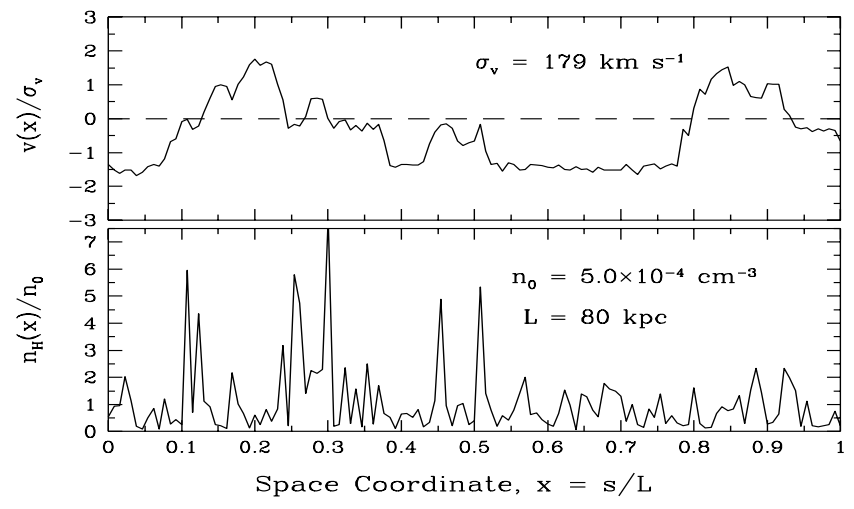

Fig. 7. Same as Fig. 5 but for the $z_{\text {abs }} \simeq 1.87$ system toward J2233-606.

\section{Discussion and conclusions}

A main goal of this work was to investigate the reliability of the physical parameters and dynamical characteristics of the metal absorption systems obtained by means of the standard Voigt fitting procedure and by the modified Monte Carlo Inversion algorithm. For comparison we used the recently obtained results on the Voigt fitting analysis of three systems toward J2233-606 (D'Odorico \& Petitjean 2001).

We found that both approaches deliver similar total column densities of unsaturated metal lines. The saturated profiles may, however, be treated differently (e.g., the Si III $\lambda 1206 \AA$ line in the $z=1.94$ system).

We also found that metal abundances based on the Voigt deconvolution procedure differ considerably from those obtained by the MCI. For instance, instead of fluctuating metallicities found in the absorbers toward J2233606 by DP, the MCI shows that an assumption of a homogeneous metal abundance for the whole system under study is quite sufficient to represent all observed features.

New and principal results which can be obtained only with the MCI procedure are the kinematic characteristics of the absorbers. We estimated selfconsistently for the first time the density and velocity dispersions along the sightlines within the absorbers and calculated the total hydrogen column and volumetric densities which gave us a direct measure of their linear sizes. The found dimensions of $L \simeq 5 \mathrm{kpc}$ to $L \simeq 80 \mathrm{kpc}$ are in good agreement with measurements of the extended gaseous envelopes around the nearby galaxes which were probed by the Mg II absorption lines (Bergeron \& Boissé 1991) and by the C IV lines (Chen et al. 2001).

A new issue obtained in the MCI analysis is a scaling relation. Namely, we found that the linear size $L$ shows a positive correlation with the line-of-sight velocity dispersion $\sigma_{\mathrm{v}}$, i.e. the higher $L$, the larger $\sigma_{\mathrm{v}}$ is observed (see Table 1). Although our sample is still too small to carry out statistical analysis of this correlation, the scaling tendency is of the same kind that can be expected for virialized systems. The velocity dispersion is closely related to the total mass of the system in a stationary state (cf. the scaling law known as the fundamental plane for elliptical galaxies). Taking into account that the scaling laws are different for different types of objects (see, e.g., Fig. 2 in Mallén-Ornelas et al. 1999), future statistical analysis may allow us to classify absorbers at different redshifts.

It is also interesting to note another scaling relation: we observe systematically higher metal abundance with decreasing $L$, and vice verse, the higher $L$, the lower metallicity is deduced. If $L$ reflects the linear size of a distant absorber, then we may conclude that a compact absorber has, presumably, higher metal content as compared with an extended one.

Acknowledgements. We thank our referee Prof. Reimers for his helpfull report. S.A.L. and I.I.A. gratefully acknowledge the hospitality of the Osservatorio Astronomico di Trieste and the National Astronomical Observatory of Japan (Mitaka) where this work was performed. We also thank Valentina D'Odorico for sharing with us the calibrated VLT/UVES spectrum of J2233-606. The work of S.A.L., I.I.A. and I.E.M. is partly supported by the RFBR grant No. 00-02-16007.

\section{Appendix A: Minimum entropy production rate}

General equation for the entropy production rate is given by (Landau \& Lifshits 1987):

$$
\begin{aligned}
& \frac{\partial S}{\partial t}=\int \frac{\kappa(\operatorname{grad} T)^{2}}{T^{2}} \mathrm{~d} V+ \\
& \int \frac{\eta}{2 T}\left(\frac{\partial v_{i}}{\partial x_{k}}+\frac{\partial v_{k}}{\partial x_{i}}-\frac{2}{3} \delta_{i k} \frac{\partial v_{j}}{\partial x_{j}}\right)^{2} \mathrm{~d} V+ \\
& \int \frac{\xi}{T}(\operatorname{div} \mathbf{v})^{2} \mathrm{~d} V
\end{aligned}
$$

Here $\partial S / \partial t$ is the rate of the total entropy production in the system, the velocity $\boldsymbol{v}$ and temperature $T$ are functions of the coordinate $(x, y, z)$, the volume element is denoted by $\mathrm{d} V$, the thermal conductivity, dynamic viscosity, and the second viscosity coefficients - by $\kappa, \eta$, and $\xi$, respectively.

The terms included in this equation account for only hydro- and thermodynamic processes since we assume the ionization balance in each point of the region which means that the radiative heating and cooling do not contribute to the entropy production. The second viscosity equals zero for dilute monoatomic gases (Chapman \& Cowling 1970) so we omit it from further consideration.

We also assume that heat conductivity $\kappa$ and viscosity $\eta$ are dominated by turbulence, and hence the Prandtl number is about unity (Monin \& Yaglom 1975):

$\operatorname{Pr}=\frac{\eta C_{\mathrm{p}}}{\kappa} \simeq 1$

where $C_{\mathrm{p}}$ is a specific heat capacity at constant pressure. The relation (A.2) is valid for both the ionized and neutral gas.

From observations, only one component $v_{x}$ (along the sightline) of the velocity vector $\boldsymbol{v}$ is known. Therefore 
Table 1. Physical parameters of the metal absorption systems toward J2233-606 derived by the MCI procedure.

\begin{tabular}{|c|c|c|c|}
\hline Parameter & $z_{\mathrm{abs}} \simeq 1.94$ & $z_{\mathrm{abs}} \simeq 1.92$ & $z_{\mathrm{abs}} \simeq 1.87$ \\
\hline Mean ionization parameter, $U_{0}$ & $8.79_{-2}(\simeq 15 \%)^{*}$ & $2.99_{-2}(\simeq 20 \%)$ & $0.12(\simeq 10 \%)$ \\
\hline Total $\mathrm{H}$ column density, $N_{\mathrm{H}}, \mathrm{cm}^{-2}$ & $1.41_{19}(\simeq 15 \%)$ & $2.69_{20}(\simeq 10 \%)$ & $1.23_{20}(\simeq 15 \%)$ \\
\hline Velocity dispersion, $\sigma_{\mathrm{v}}, \mathrm{km} \mathrm{s}^{-1}$ & $35(\simeq 15 \%)$ & $104(\simeq 20 \%)$ & $179(\simeq 10 \%)$ \\
\hline Density dispersion, $\sigma_{\mathrm{y}}$ & $1.50(\simeq 15 \%)$ & $2.31(\simeq 10 \%)$ & $1.31(\simeq 10 \%)$ \\
\hline \multicolumn{4}{|l|}{ Chemical abundances $^{\dagger}$ : } \\
\hline$Z_{\mathrm{C}}$ & $9.8_{-5}(\lesssim 10 \%)$ & $2.0_{-6}(\simeq 20 \%)$ & $8.7_{-6}(\lesssim 10 \%)$ \\
\hline$Z_{\mathrm{N}}$ & $1.1_{-5}(\simeq 15 \%)$ & $5.5_{-7}(\simeq 20 \%)$ & $\ldots$ \\
\hline$Z_{\mathrm{Mg}}$ & $6.8_{-6}(\simeq 10 \%)$ & $\ldots$ & $2.0_{-6}(\lesssim 10 \%)$ \\
\hline$Z_{\mathrm{Al}}$ & $5.1_{-7}(\simeq 15 \%)$ & $\ldots$ & $\ldots$ \\
\hline$Z_{\mathrm{Si}}$ & $1.0_{-5}(\lesssim 10 \%)$ & $2.5_{-7}(\simeq 20 \%)$ & $1.7_{-6}(\lesssim 10 \%)$ \\
\hline$\left[Z_{\mathrm{C}}\right]$ & -0.53 & -2.21 & -1.58 \\
\hline$\left[Z_{\mathrm{N}}\right]$ & -0.88 & -2.17 & $\ldots$ \\
\hline$\left[Z_{\mathrm{Mg}}\right]$ & -0.75 & $\ldots$ & -1.27 \\
\hline$\left[Z_{\mathrm{Al}}\right]$ & -0.79 & $\ldots$ & $\ldots$ \\
\hline$\left[Z_{\mathrm{Si}}\right]$ & -0.54 & -2.14 & -1.31 \\
\hline \multicolumn{4}{|l|}{ Column densities, $\mathrm{cm}^{-2}$ : } \\
\hline$N(\mathrm{H} \mathrm{I})$ & $(2.50 \pm 0.05)_{16}$ & $(2.2 \pm 0.25)_{17}$ & $(2.65 \pm 0.28)_{16}$ \\
\hline$N(\mathrm{C}$ II $)$ & $(7.49 \pm 0.11)_{13}$ & $(1.80 \pm 0.20)_{13}$ & $\ldots$ \\
\hline$N(\mathrm{Mg}$ II $)$ & $(6.04 \pm 0.22)_{12}$ & $\ldots$ & $(1.96 \pm 0.21)_{12}$ \\
\hline$N(\mathrm{Si}$ II $)$ & $(8.79 \pm 0.25)_{12}$ & $(2.40 \pm 0.16)_{12}$ & $(2.56 \pm 0.20)_{12}$ \\
\hline$N(\mathrm{C}$ III $)$ & $\ldots$ & $\ldots$ & $(5.36 \pm 0.25)_{14}$ \\
\hline$N(\mathrm{~N}$ ІіI $)$ & $(1.62 \pm 0.09)_{14}$ & $\ldots$ & $\ldots$ \\
\hline$N(\mathrm{Al}$ III $)$ & $(1.63 \pm 0.08)_{12}$ & $\ldots$ & $\ldots$ \\
\hline$N($ Si III $)$ & $(9.33 \pm 0.51)_{13}$ & $(2.90 \pm 0.18)_{13}$ & $(4.94 \pm 0.25)_{13}$ \\
\hline$N(\mathrm{C}$ IV $)$ & $(3.93 \pm 0.16)_{14}$ & $(4.78 \pm 0.31)_{13}$ & $(3.66 \pm 0.13)_{14}$ \\
\hline$N(\mathrm{Si}$ IV $)$ & $(5.58 \pm 0.35)_{13}$ & $(8.50 \pm 0.50)_{12}$ & $(4.13 \pm 0.11)_{13}$ \\
\hline$N(\mathrm{~N} \mathrm{~V})$ & $<3.54_{12}$ & $\ldots$ & $\ldots$ \\
\hline Hydrogen number density, $n_{0}, \mathrm{~cm}^{-3}$ & $\simeq 1_{-3}$ & $\simeq 5_{-3}$ & $\simeq 5_{-4}$ \\
\hline Mean kinetic temperature, $\mathrm{K}$ & $14_{3}$ & $25_{3}$ & $25_{3}$ \\
\hline Minimum kinetic temperature, $\mathrm{K}$ & $11_{3}$ & $16_{3}$ & $18_{3}$ \\
\hline Maximum kinetic temperature, $\mathrm{K}$ & $17_{3}$ & $40_{3}$ & $31.5_{3}$ \\
\hline Linear size, $L, \mathrm{kpc}$ & $\simeq 5$ & $\simeq 20$ & $\simeq 77$ \\
\hline Mass, $M^{\ddagger}$, in $M_{\odot}$ & $\simeq 1.4_{6}$ & $\simeq 4.5_{8}$ & $\simeq 2.6_{9}$ \\
\hline Mass, $M^{\diamond}$, in $M_{\odot}$ & $\simeq 7.0_{8}$ & $\simeq 2.0_{10}$ & $\simeq 3.0_{11}$ \\
\hline
\end{tabular}

*Values like " $a_{x}$ " mean $a \times 10^{x}$; shown in parenthesis are uncertainties at the $1 \sigma$ c.l.

${ }^{\dagger} Z_{\mathrm{X}}=\mathrm{X} / \mathrm{H},\left[Z_{\mathrm{X}}\right]=\log \left(Z_{\mathrm{X}}\right)-\log \left(Z_{\mathrm{X}}\right) \odot$.

${ }^{\ddagger}$ Assuming spherical geometry; ${ }^{\diamond}$ Using the virial theorem.

we are compelled to neglect in (A.1) all terms including derivatives other than $\partial v_{x} / \partial x$. The second right hand term in (A.1) can be re-written in the form:

$\int \frac{\eta}{2 T}\left(\frac{\partial v_{i}}{\partial x_{k}}+\frac{\partial v_{k}}{\partial x_{i}}-\frac{2}{3} \delta_{i k} \frac{\partial v_{j}}{\partial x_{j}}\right)^{2} \mathrm{~d} V=$
$\int \frac{\eta}{2 T}\left(\frac{\partial v_{i}}{\partial x_{k}}+\frac{\partial v_{k}}{\partial x_{i}}\right)^{2} \mathrm{~d} V-\frac{4}{3} \int \frac{\eta}{2 T}(\operatorname{div} \boldsymbol{v})^{2} \mathrm{~d} V$

After all these assumptions, we obtain the following simplified 1D form for the entropy production rate

$$
\begin{aligned}
& \frac{\partial \tilde{S}}{\partial t}= \\
& \int \kappa\left[\frac{1}{\tilde{T}^{2}}\left(\frac{\mathrm{d} \tilde{T}}{\mathrm{~d} x}\right)^{2}+\operatorname{Pr} \frac{\sigma_{\mathrm{v}}^{2}}{C_{\mathrm{p}} T_{0}} \frac{\mathcal{A}}{\tilde{T}}\left(\frac{\mathrm{d} \tilde{v}_{x}}{\mathrm{~d} x}\right)^{2}\right] \mathrm{d} x,
\end{aligned}
$$

where $\tilde{v}_{x}=v_{x} / \sigma_{\mathrm{v}}, \tilde{T}=T / T_{0}$, with $\sigma_{\mathrm{v}}$ and $T_{0}$ being scales (characteristic values) for velocity and temperature inside the considered region, $\mathcal{A}$ is a constant of about 1 , and $\tilde{S}$ is the entropy per unit area.

For a monatomic ideal gas $C_{\mathrm{p}}=5 n R / 2(n$ is the number of gram-moles and $R$ is the universal gas constant), and if the gas is fully ionized then $\kappa \propto T^{5 / 2}$ (e.g. Lang 1999).

Given the values of $\kappa, \operatorname{Pr}, T_{0}, C_{\mathrm{p}}$, and $\sigma_{\mathrm{v}}$, we can rearrange the computational points $\left\{v_{1}, \ldots, v_{k}\right\}$ and $\left\{n_{1}, \ldots, n_{k}\right\}$ in such a manner, that minimum of (A.4) will be achieved. Minimization of (A.4) was carried out by means of combinatorial simulated annealing technique (Press et al. 1992). The obtained configuration of the density and velocity distributions should have the least dissipation and therefore will exist longer than all others. 


\section{References}

Bergeron, J., \& Boissé, P. 1991, A\&A, 243, 344

Chapman, S., \& Cowling, T. G. 1970, The Mathematical Theory of Non-Uniform Gases, 3rd ed. (Cambridge Univ. Press: Cambridge)

Chen, H.-W., Lanzetta, K. M., Webb, J. K., \& Barcons, X. 1998, ApJ, 498, 77

Chen, H.-W., Lanzetta, K. M., \& Webb, J. K. 2001, ApJ, 556, 158

Cristiani, S., \& D'Odorico, V. 2000, AJ, 120, 1648

D'Odorico, V., \& Petitjean, P. 2001, A\&A, 370, 729 (DP)

Ferland, G. J. 1997, Hazy, a brief introduction to Cloudy 94.00 (http://www.pa.uky.edu/ gary/cloudy)

Giallongo, E., \& Petitjean, P. 1994, ApJ, 426, L61

Haardt, F., \& Madau, P. 1996, ApJ, 461, 20

Kirkman, D. K., \& Tytler, D. 1999, ApJ, 512, L5

Landau, L. D., \& Lifshitz, E. M. 1987, Fluid Mechanics (Butterworth-Heinemann)

Lang, K. R. 1999, Astrophysical Formulae, vol. 1 (SpringerVerlag: Berlin Heidelberg)

Levshakov, S. A., Takahara, F., \& Agafonova, I. I. 1999, ApJ, 517,609

Levshakov, S. A., Agafonova, I. I. , \& Kegel, W. H. 2000a, A\&A, 355, L1
Levshakov, S. A., Agafonova, I. I. , \& Kegel, W. H. 2000b, A\&A, 360, 833, Paper I

Levshakov, S. A., Dessauges-Zavadsky, M., D'Odorico, S., \& Molaro, P. 2002, ApJ, 565, 696

Mallén-Ornelas, G., Lilly, S. J., Crampton, D., \& Schade, D. 1999, ApJ, 518, L83

Monin, A. S., \& Yaglom, A. M. 1975, Statistical Fluid Mechanics: Mechanics of Turbulence, vol. 2 (Cambridge: MIT)

Press, W. H., Teukolsky, S. A., Vetterling, W. T., \& Flannery, B. P. 1992, Numerical Recipes (Cambridge Univ. Press: Cambridge)

Prigogine, I. 1967, Thermodynamics of Irreversible Processes (Wiley: New York)

Prochaska, J. K., \& Burles, S. M. 1999, AJ, 117, 1957

Reimers, D., Baade, R., Hagen, H.-J., \& Lopez, S. 2001, A\&A, 374,871

Savaglio, S. 1998, AJ, 116, 1055

Savin, D. W. 2000, ApJ, 533, 106

Viegas, S. M., Friaca, A. C. S., \& Gruenwald, R. 1999, MNRAS, 309, 355

Xiang, Y., Sun, D. Y., Fan, W., \& Gong, X. G. 1997, Phys. Lett. A, 233, 216 